Acta Crystallographica Section D

Biological

Crystallography

ISSN 0907-4449

\title{
Distributed structure determination at the JCSG
}

The Joint Center for Structural Genomics (JCSG), one of four large-scale structure-determination centers funded by the US Protein Structure Initiative (PSI) through the National Institute for General Medical Sciences, has been operating an automated distributed structure-solution pipeline, Xsolve, for well over half a decade. During PSI-2, Xsolve solved, traced and partially refined $90 \%$ of the JCSG's nearly $770 \mathrm{MAD} /$ SAD structures at an average resolution of about $2 \AA$ without human intervention. Xsolve executes many well established publicly available crystallography software programs in parallel on a commodity Linux cluster, resulting in multiple traces for any given target. Additional software programs have been developed and integrated into Xsolve to further minimize human effort in structure refinement. ConsensusModeler exploits complementarities in traces from Xsolve to compute a single optimal model for manual refinement. Xpleo is a powerful robotics-inspired algorithm to build missing fragments and qFit automatically identifies and fits alternate conformations.

\section{Introduction}

The Protein Structure Initiative is a national effort in the USA to determine a large collection of three-dimensional protein structures in a high-throughput operation, with the long-term objective of making the atomic level details of most proteins easily obtainable from their corresponding DNA sequences. In the pilot phase, which ran from 2000 to 2005, new approaches and tools were developed to streamline and automate the steps of protein structure determination. In the subsequent production phase, which ended in mid-2010, these high-throughput methods resulted in a large number of unique protein structures. Since its inception in 2000, the Joint Center for Structural Genomics (JCSG) has focused on the development of methodologies and protocols to automate and streamline its structural genomics process, from target selection and protein expression to ultimately the deposition of high-quality three-dimensional structures in the Protein Data Bank (PDB; Berman et al., 2003).

Here, we present the software suite Xsolve, which was developed by the JCSG to automatically execute all steps of the X-ray structure-determination process from reading diffraction images to calculating a partially refined threedimensional model and thus reduce the need for human intervention. Fully automating routine tasks associated with solving a structure allows the JCSG to focus its efforts on more demanding aspects of structure determination, ensuring that a high-quality structure will be produced. In contrast to pre-
Received 30 July 2010 Accepted 6 October 2010 
viously reported automated structure-determination methods (Holton \& Alber, 2004; Fu et al., 2005; Panjikar et al., 2005; Adams et al., 2010), Xsolve was designed to explore key parameters of protein structure determination independently and in parallel across all stages of the process. It also employs multiple software programs for identical tasks at each stage. Many 'promising' parameter and program combinations are simultaneously carried forward to the final stages, resulting in many structures with varying degrees of completeness and accuracy. While many processing strategies lead to good protein structures, there are numerous cases in which some strategies fail or are substantially outperformed by others. All final structures are then collected by ConsensusModeler, which exploits their complementarities by computing a consensus model that serves as an optimal starting point for subsequent manual refinement.

\section{Methods}

Xsolve's design reflects a distributed data-driven approach to solving protein structures; rather than trying to execute a single best strategy to arrive at an optimal set of initial coordinates (a 'trace') for further refinement, it explores many independent trials in parallel. Xsolve computes a 'tree' of solutions, with the traces (the leaves of the tree) being the end result of a long sequence of branch points throughout the structure-determination process. The distributed tasks are then reduced into a final trace. This general architecture, which is commonly used in modern computing, is highly robust to failure from suboptimal processing or hardware malfunction.

\subsection{Xsolve}

Xsolve implements all processing steps required to compute an electron-density map and a trace from diffraction images. It executes well established third-party software programs for data reduction (indexing, integration and scaling), phasing and tracing of the experimental maps in succession without human

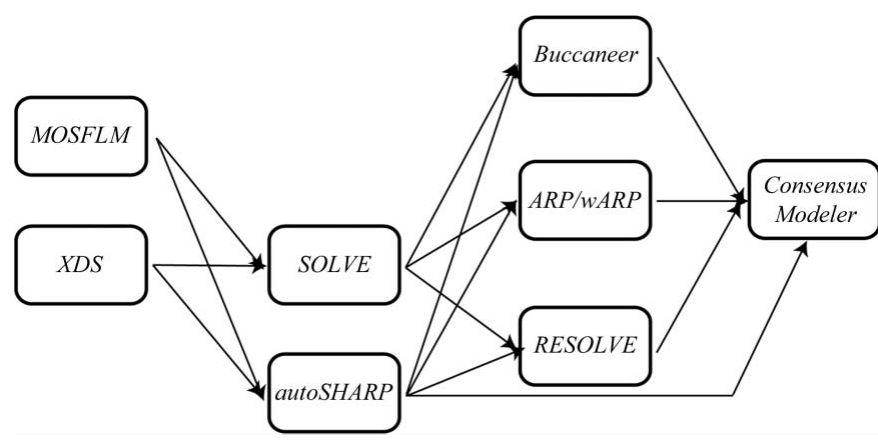

Figure 1

Parallelization at the 'program level' in Xsolve. All outputs at each stage of $X$ solve are distributed independently and in parallel to all programs at the next stage. Shown here are 14 combinations of software programs at the three stages in structure determination. autoSHARP includes model building with $A R P / w A R P$ and the resulting models are collected by ConsensusModeler. autoSHARP phases are input to Buccaneer and RESOLVE. intervention. The third-party software programs currently included are MOSFLM (Leslie, 1992), XDS (Kabsch, 2010) and $H K L-2000$ (Otwinowski \& Minor, 1997; for testing only) for data reduction; SHELXD (Sheldrick, 2008), SOLVE (Terwilliger \& Berendzen, 1999) and autoSHARP (Vonrhein et al., 2007) for heavy-atom location and phasing; and ARP/ wARP (Perrakis et al., 1999), RESOLVE (Terwilliger, 2003) and Buccaneer (Cowtan, 2006) for building a model into the electron-density map. Fig. 1 depicts the flow of information in Xsolve.

Xsolve can also solve a data set using molecular replacement (MR). A protocol similar to that reported in Schwarzenbacher et al. (2008) was implemented. Parameters such as multiple MR templates, resolution cutoff and space groups are explored in parallel using multiple MR programs: MOLREP (Vagin \& Teplyakov, 2010), EPMR (Kissinger et al., 1999) and Phaser (Storoni et al., 2004). Potential MR solutions are subjected to rigid-body refinement and restrained refinement with REFMAC (Murshudov et al., 1997). Model rebuilding is carried out using $A R P / w A R P$ and RESOLVE. As the JCSG uses single-wavelength or multiwavelength anomalous diffraction (SAD/MAD) techniques to obtain phases for the vast majority of its targets, the remainder of this paper will focus on SAD/MAD data.

Once a data set has been collected, a crystallographer completes a simple web form to inform the system of a few parameters, such as the location of the diffraction images, the resolution limit and theoretical or experimentally determined anomalous scattering factors. Optionally, to limit the search space, space groups and the number of monomers in the asymmetric unit can be provided as input to Xsolve. The datacollection strategy is reconstructed from parsing diffractionimage headers. Additional parameters, such as the amino-acid sequence, molecular weight and heavy-atom information, are automatically read from a database. The molecular weight can also trivially be derived from the sequence. Once this is complete, the job can be submitted to Xsolve with a different web form. The status of Xsolve can also be checked within a browser. Screenshots of the three web forms are included as supplementary material ${ }^{\mathbf{1}}$ to this paper.

2.1.1. Parallelization. Independent trials are executed in parallel on a 300-core compute cluster. Parallelism is employed at the 'data level' and at the 'program level'. At the 'data level' multiple space groups, (MAD) wavelength combinations and number of monomers per asymmetric unit are sampled. For instance, a crystallographer can instruct $X$ solve to solve the structure in space groups $P 2_{1}$ and $P 2_{1} 2_{1} 2_{1}$ to account for possible higher metric symmetry. Wildcards are also accepted, so that $P 2 *$ will be expanded by $X$ solve. Unless explicitly overridden, Xsolve will attempt to solve the structure by sampling a number of monomers per asymmetric unit compatible with the estimated solvent content of the unit cell. The JCSG typically collects MAD data at three wavelengths

\footnotetext{
${ }^{\mathbf{1}}$ Supplementary material has been deposited in the IUCr electronic archive (Reference: BA5156). Services for accessing this material are described at the back of the journal.
} 
whenever possible. All wavelength combinations are explored simultaneously from the data-reduction step on to evaluate the best phases independent of possible radiation damage to the crystal. Similarly, at the 'program level' all combinations of the third-party software programs are explored in parallel. For instance, whenever a wavelength/space-group combination is output at the integration stage, it serves as input to all programs at the phasing stage. When one of the phasing applications outputs an experimental map corresponding to a wavelength/space group/monomer combination it serves as input to all model-building programs. Fig. 1 displays 14 combinations of software programs at the three major stages of structure determination. Assuming a fixed choice of space group and a fixed number of monomers per asymmetric unit, together with six wavelength combinations, this already leads to 72 processing strategies. In practice, many strategies are easily determined to be suboptimal and are pruned at an early stage. The resulting traces are ultimately collected by ConsensusModeler and condensed into a single optimal trace.

2.1.2. Implementation. Xsolve was implemented in Java following a master/worker model. Each compute core of the cluster is associated with a worker module that communicates with a central 'master' server module. The server generates jobs, which it holds in a queue for the worker nodes to process. The third-party software programs are started from shell scripts that are generated dynamically at each stage. These shell scripts are generally very simple, consisting of a call of the program for the next processing step together with the input parameters that were determined in the preceding processing steps. Information flows from one program to the next by means of Extensible Markup Language (XML) intermediary format files (W3C World Wide Web Consortium; http://www.w3.org/XML/). Upon the successful completion of a worker task, the Java execution environment parses the task $\log$ file and stores the values of pertinent parameters in XML format. The correct parameters are then imported into the next task's shell script. This XML-driven architecture facilitates manual intervention at any stage, if desired, and in addition completely decouples job scheduling and execution from the crystallographic workflow; new processing stages are easily added by modifying a shell script template.

\subsection{ConsensusModeler: combining traces}

ConsensusModeler capitalizes on Xsolve's exploration of model building by combining traces output by different strategies to obtain a more complete and error-corrected trace. ConsensusModeler collects Xsolve traces that have more than $40 \%$ of side chains docked into the density. Trace errors for models not meeting this threshold tend to be severe. The ConsensusModeler algorithm first superimposes traces using crystallographic symmetry operators, automatically shifting the origin or re-indexing the data wherever necessary. Next, NCS-related traces are superimposed using SSM (Krissinel \& Henrick, 2004). The ConsensusModeler algorithm accepts the sequence (side-chain identity) assignment from each of its input traces; any conflicts will be, by its nature, optimally resolved by the algorithm. Undocked fragments are set aside. Each superimposed trace is represented in a graph, with each residue corresponding to a vertex (Fig. 2). Vertices (residues) from all contributing input traces are connected by directed edges such that a residue with sequence number $i-1$ from any subunit in any trace $j$ is connected to all residues with sequence number $i$ in all subunits of all traces. Each edge is assigned a score or 'weight' to reflect how its pair of residues fits the electron density and how the pair would affect the quality of the final model. The calculation of edge weights is detailed in the next section. Once edge weights have been determined, the Bellman-Ford algorithm (Heineman et al., 2008) is executed from the $\mathrm{N}$-terminus to the $\mathrm{C}$-terminus and from the $\mathrm{C}$-terminus to the $\mathrm{N}$-terminus to find a path through the graph that minimizes the total weight. The trace that corresponds to the path of minimum weight is output. ConsensusModeler was implemented in $\mathrm{C}++$ and uses the Clipper libraries (Cowtan, 2000) for crystallographic computations.

2.2.1. Edge weights. Edge weights are heuristically derived values designed to identify and reward favorable features in a trace and penalize unfavorable features or errors such as mistracings and 'frame-shifts'. The following features are taken into account.

(i) Agreement with the electron density. For each residue, agreement with the electron density is measured with a density cross-correlation coefficient computed by the algorithm.

(ii) Agreement with other residues. If other input traces have the same residue modeled at a spatial position, it is more likely to be correct than in cases where other traces have a different residue modeled. The number of similar residues at a single spatial position inversely contributes to the edge weight.

(iii) Geometry penalties. A penalty is incurred for disallowed Ramachandran values or whenever the distance of subsequent $\mathrm{C}^{\alpha}$ atoms substantially deviates from the mean inter-peptide value.

(iv) Overlap penalty. Incorrectly traced backbones by model-building programs may result in multiple residues with distinct sequence numbers occupying the same spatial location after superposition. While the edge weight described under (ii) above increases the likelihood that the correct residue is

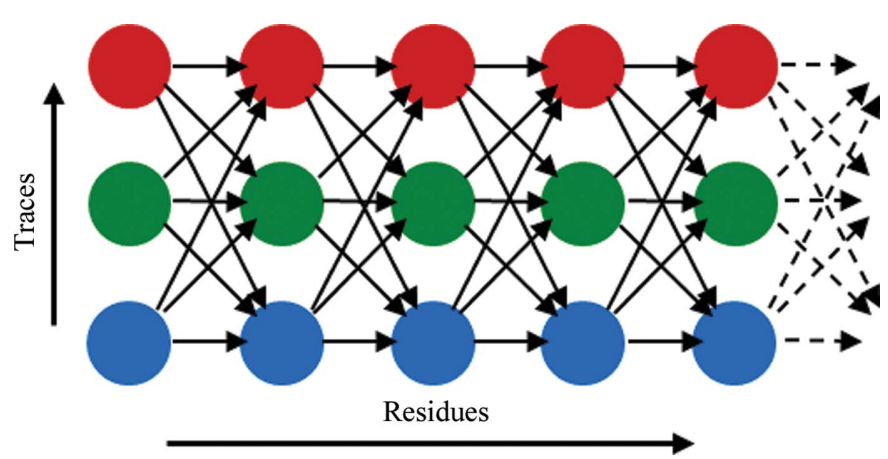

Figure 2

Superimposed traces represented as a graph. A docked trace is represented from left to right; traces resulting from different modelbuilding protocols are represented on the vertical axis. 
inserted at the correct sequence position in the consensus model, it does not prevent an incorrectly traced residue at this spatial location also being included at its sequence position. This penalty aims to make overlapping residues mutually exclusive.

(v) Gaps. Analogous to sequence-alignment algorithms (e.g. Smith \& Waterman, 1981), an output model can have gaps, i.e. missing fragments. Such a situation could arise if none of the input models have a residue modeled for the sequence position (often at the termini) or if any residue from the input models would result in an output model with higher score than a gap would. Gaps are modeled with 'dummy' residues. There is a high one-time gap-opening penalty and furthermore a lower penalty for each dummy residue to continue a gap.

2.2.2. Xpleo. The fragment-fitting software program Xpleo (available from $\mathrm{http} / / / \mathrm{smb}$.slac.stanford.edu/ vdbedem; van den Bedem et al., 2005) was integrated with ConsensusModeler. Gaps of fewer than 15 residues in length in the consensus model are automatically identified, built and included.

2.2.3. qFit. The JCSG has recently developed an integer quadratic programming-based algorithm qFit (van den Bedem et al., 2009) to identify and model alternate side-chain and main-chain conformations together with their occupancies. The software reduces subjectivity in assigning alternate conformations and the resulting models show an improvement in the $R_{\text {free }}$ statistic (Brünger, 1992). Work is under way to integrate qFit into Xsolve.

\section{Results}

Xsolve was integrated into the JCSG structure-determination and refinement workflow. As part of the routine interaction with Xsolve, at termination the best traces are visually inspected by one of the JCSG's staff crystallographers. Among these traces, one is selected to be uploaded to the JCSG's tracking database to aid in further refinement. Generally, the longest trace with correctly docked side chains, i.e. the most complete trace, is selected.

By early 2010, nearly 770 structures had been deposited in the PDB. In $70 \%$ of cases an initial trace obtained from $A R P / w A R P$ was selected at a mean resolution of $1.8 \AA$. The remaining $30 \%$ were traced by $R E S O L V E^{2}$ at a mean resolution of $2.1 \AA$. For the high-resolution quartile of data sets solved to better than $1.7 \AA$, ARP/wARP contributed $83 \%$ of the best traces, with RESOLVE contributing the remaining $17 \%$. For the low-resolution quartile, i.e. data sets solved to worse than $2.1 \AA$, these proportions were 69 and $31 \%$, respectively. The mean solvent contents were nearly identical at 49.8 and $50.7 \%$, respectively. As expected, owing to the difference in mean resolution, the mean $R_{\text {free }}$ for the $A R P /$ $w A R P$ traces was slightly lower, at 0.205 versus 0.227 .

Descriptive statistics were also computed for phasing software programs. It was found that $72 \%$ of the uploaded traces were phased with $S H A R P$ at a mean resolution of $1.88 \AA$ and

\footnotetext{
${ }^{2}$ Buccaneer was added to Xsolve early in 2010 and has been excluded from the analysis.
}

Table 1

Characteristics of the 36 data sets selected for reprocessing.

The information in this table may differ from that displayed in the PDB archive. For some targets data were collected from an additional crystal to facilitate refinement.

\begin{tabular}{|c|c|c|c|c|}
\hline PDB code & $\begin{array}{l}\text { Space } \\
\text { group }\end{array}$ & $\begin{array}{l}\text { Sequence } \\
\text { length }\end{array}$ & $\begin{array}{l}\text { Molecules in } \\
\text { asymmetric unit }\end{array}$ & $\begin{array}{l}\text { Resolution } \\
(\AA)\end{array}$ \\
\hline $3 \mathrm{mcp}$ & $P 4_{3} 2_{1} 2$ & 365 & 1 & 3.0 \\
\hline $3 \mathrm{n} 0 \mathrm{v}$ & $P 2_{1}$ & 285 & 4 & 2.89 \\
\hline $3 \mathrm{ksr}$ & $P 4_{3} 2_{1} 2$ & 289 & 1 & 2.8 \\
\hline $3 e c 4$ & $P 2_{1} 2_{1} 2_{1}$ & 227 & 2 & 2.8 \\
\hline $3 \mathrm{k} 8 \mathrm{r}$ & $I 4$ & 123 & 2 & 2.75 \\
\hline $3 \mathrm{cuc}$ & $P 3_{1} 21$ & 290 & 2 & 2.71 \\
\hline $3 \mathrm{k} 9 \mathrm{i}$ & $P 6_{1} 22$ & 117 & 1 & 2.71 \\
\hline $3 \operatorname{do} 5$ & $C 222_{1}$ & 326 & 1 & 2.7 \\
\hline $3 b j q$ & $P 2_{1}$ & 315 & 10 & 2.6 \\
\hline $2 \mathrm{qdr}$ & $P 6_{2} 22$ & 302 & 2 & 2.6 \\
\hline $3 \mathrm{dxq}$ & $P 2_{1} 2_{1} 2$ & 300 & 2 & 2.55 \\
\hline $2 \mathrm{re} 3$ & $P 4_{3}{ }_{1} 2$ & 193 & 2 & 2.53 \\
\hline YP_001197814.1 & $P 6_{1} 22$ & 236 & 1 & 2.5 \\
\hline $3 \mathrm{knz}$ & $P 2_{1} 2_{1} 2_{1}$ & 347 & 6 & 2.5 \\
\hline $3 \mathrm{~d} 1 \mathrm{c}$ & $P 4_{3} 2_{1} 2$ & 368 & 1 & 2.4 \\
\hline 2op5 & $P 2_{1} 2_{1} 2_{1}$ & 116 & 6 & 2.35 \\
\hline $3 \mathrm{dxp}$ & $P 2_{1} 2_{1} 2$ & 358 & 1 & 2.32 \\
\hline 3dde & $P 2_{1} 22_{1}$ & 238 & 2 & 2.3 \\
\hline $3 d 7 q$ & $P 4_{3}{ }_{1} 2$ & 111 & 2 & 2.3 \\
\hline $3 \mathrm{dkq}$ & $P 4_{1} 2_{1} 2$ & 224 & 3 & 2.26 \\
\hline NP_388303.1 & $P 2_{1} 2_{1} 2_{1}$ & 140 & 1 & 2.06 \\
\hline $3 \mathrm{k} 6 \mathrm{o}$ & $C 2$ & 223 & 2 & 2 \\
\hline $3 \mathrm{htv}$ & $C 222_{1}$ & 309 & 1 & 1.95 \\
\hline 3gyc & $P 2_{1}$ & 392 & 2 & 1.85 \\
\hline $3 \mathrm{kog}$ & $I 2_{1} 2_{1} 2_{1}$ & 255 & 1 & 1.85 \\
\hline $3 \mathrm{fcr}$ & $C 2$ & 457 & 1 & 1.8 \\
\hline $2 \mathrm{p} 7 \mathrm{i}$ & $I 422$ & 249 & 2 & 1.75 \\
\hline $2 \mathrm{pfx}$ & $P 6_{3}$ & 190 & 2 & 1.7 \\
\hline 2qeu & $P 6_{1} 22$ & 140 & 3 & 1.65 \\
\hline $20 u 5$ & $P 2_{1} 2_{1} 2_{1}$ & 174 & 2 & 1.6 \\
\hline $3 f 8 x$ & $P 1$ & 147 & 4 & 1.55 \\
\hline $3 h d x$ & $P 4_{1}{ }_{1} 2$ & 477 & 1 & 1.5 \\
\hline $3 g r 3$ & $P 2_{1} 2_{1} 2_{1}$ & 229 & 2 & 1.45 \\
\hline 3 is $x$ & $C 2$ & 331 & 1 & 1.4 \\
\hline 2qjw & $P 2_{1}$ & 175 & 4 & 1.35 \\
\hline 3hwu & $H 3$ & 146 & 1 & 1.3 \\
\hline
\end{tabular}

the remaining $28 \%$ were phased with SOLVE (mean resolution $1.91 \AA$ ). One out of three RESOLVE traces and one out of four ARP/wARP traces were phased with SOLVE. The slightly higher fraction for RESOLVE is possibly explained by these programs originating from the same author. The subsets of SOLVE- and SHARP-phased traces within the RESOLVE and $A R P / w A R P$ trace sets had identical mean resolutions, i.e. resolution outweighs the choice of phasing program for the efficacy of model-building programs.

The parallel exploration of parameters and processing strategies by Xsolve was particularly advantageous in solving challenging data sets. For instance, the JCSG solved 16 structures from twinned data sets using the MAD/SAD method, with twin fractions ranging from 0.14 to 0.48 (PDB entries 2i5i, 2p4g, 2pfw, 2pfx, 2prx, 2pyq, 2q02, 2q22, 3db2, $3 \mathrm{duk}$, 3ejn, 3fxa, 3kst, 3mc3, 3b9t and 3lws). In these cases, traces were obtained from a solution in the apparent higher order space group as well as the correct space group and different phasing/density-modification/tracing program combinations. In many instances structures were solved in both space groups, but often initial traces were better in the higher 


\section{Table 2}

Average percentage of side chains docked into electron density for each of the three model-building programs and the number of data sets for which it produced the most complete trace.

The average was calculated over all models that had $40 \%$ or more of side chains docked.

\begin{tabular}{|c|c|c|c|}
\hline & $A R P / w A R P$ & RESOLVE & Buccaneer \\
\hline All resolutions ( $\%)$ & 84.7 & 72.7 & 89.5 \\
\hline Better than $1.8 \AA$ & 87.1 & 83.3 & 89.2 \\
\hline Worse than $2.5 \AA(\%)$ & 0 & 60.1 & 87.2 \\
\hline No. of top traces & 2 & 1 & 33 \\
\hline
\end{tabular}

space group. Similarly, Xsolve has allowed the JCSG to evaluate multiple solutions when the initial space group is ambiguous.

\subsection{Insights from reprocessing 36 data sets}

36 previously solved data sets were selected across various resolutions, space groups and sizes (Table 1).

Fig. 3 graphically represents the completeness of traces resulting from distinct data-processing strategies in Xsolve. While the numbers of output traces are only shown for the correct content of the asymmetric unit and are summarized over wavelength combinations, the figure bears out that Xsolve's volume of output exceeds what can efficiently be visually inspected in a high-throughput production environment. In 33 out of 36 cases (92\%) Buccaneer reported the most complete trace. $A R P / w A R P$ traces, shown in the foreground, exhibit a high degree of completeness at higher resolutions. For the seven data sets at a resolution of $1.6 \AA$ or better, two of the most complete traces resulted from $A R P / w A R P$, one from RESOLVE and the remainder from Buccaneer. At lower resolution, RESOLVE (mid-section) and Buccaneer (background) provide traces that are more complete than those of ARP/wARP (Table 2).

Particularly successful was the combination $X D S / S H A R P /$ Buccaneer, which accounted for $42 \%$ of all top traces (the greatest proportion of residues docked reported) across all resolutions (Fig. 4). However, all indexing, phasing and modelbuilding software programs are represented among the top traces. Qualitatively, it was observed that while Buccaneer reports more complete traces, its error rate, i.e. fragments incorrectly docked into the density, tends to be higher than RESOLVE's at lower resolution. Buccaneer is also fast, in one case tracing more than 3000 residues to $92 \%$ completeness in 90 min (PDB entry 3bjq; Table 1).

In Xsolve's design, more important than a strategy's ability to produce a top trace is simply that it differs from others and thus contains additional information. Manually evaluating each trace would be prohibitively labor-intensive, as Xsolve can produce dozens. ConsensusModeler capitalizes on the divergence in accuracy and completeness of input traces and computes a trace that is better than any input.

Overall, ConsensusModeler provided a modest average improvement to the longest reported trace of about $1 \%$
- buccaneer_sharp_mosflm mautowarp_sharp_mosflm_phaseres warp_solve_xds a resolve_sharp_mosflm muccaneer_solve_xds -1 resolve_sharp_xds warp_sharp_xds additional residues docked into the electron density. Improvements in completeness are balanced by error correction of more aggressive traces. One in six traces improved more than 5\% (Fig. 5), with a maximum of $38 \%$. Indeed, occasionally traces that report lower completeness contain highly complementary parts. The crystal structure of a putative serine hydrolase (NP_639225.1) from Xanthomonas campestris was solved at $2.8 \AA$ resolution (PDB entry 3ksr). In this case, Xsolve output only two traces: a Buccaneer trace with $57 \%$ of the residues docked and a RESOLVE trace with $39 \%$ of the residues docked. These highly complementary traces were combined by ConsensusModeler into a trace that was $78 \%$ complete (Fig. 6).

Five of the 36 consensus models had fewer than $95 \%$ of the residues docked compared with the best trace (Fig. 5). Of these five, two have not resulted 
in a PDB deposition as none of the Xsolve traces or consensus models were deemed sufficiently complete and accurate to proceed with refinement (YP_001197814.1 and NP_388303.1). Closer examination of the other three revealed that in one case ConsensusModeler had successfully omitted two incorrectly traced long fragments (in $3 \mathrm{mcp}$ ), while in others it had erroneously removed a helix (in $3 \mathrm{k} 9 \mathrm{i}$ ) and a 20 -residue fragment (in 3dkq).

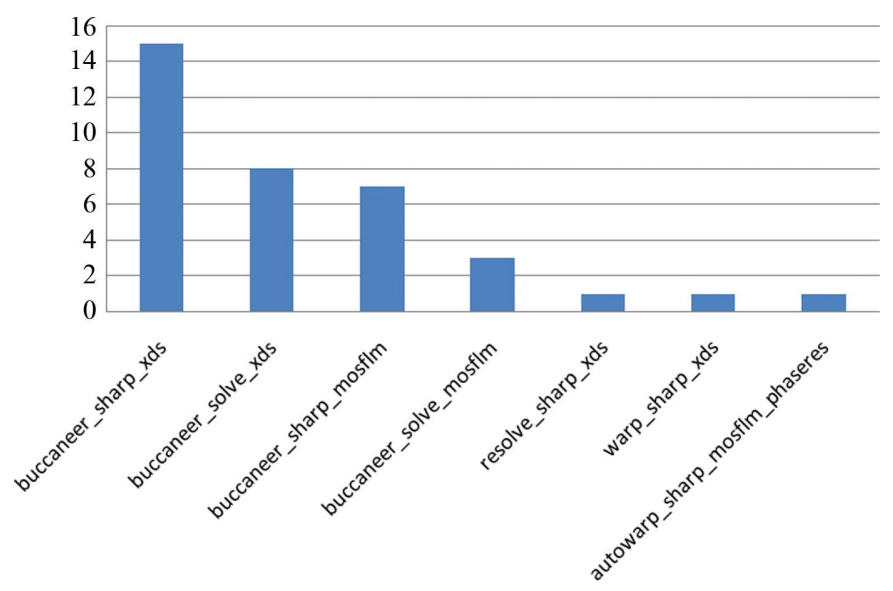

Figure 4

Number of times an indexing, phasing and model-building combination contributed the top trace for the 36 targets.

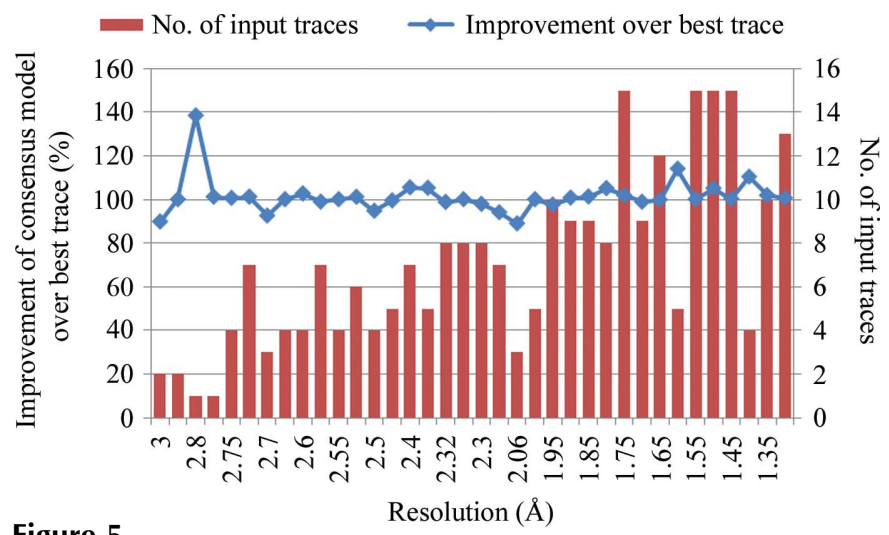

Figure 5

Percentage of improvement of the number of side chains docked to the electron density by the consensus model over the best input trace (blue line, left axis). The bars depict the number of input traces to ConsensusModeler. Only input traces with the correct space group and number of molecules in the asymmetric unit were considered. Wavelength combinations were binned, so that one input trace is reported for each program combination, similar to Fig. 3.
ConsensusModeler also facilitated Xpleo by partially closing gaps in input traces. The remaining missing fragments were easily computed and fitted to the electron density with Xpleo. The structure of a putative oxygenase (YP_001051978.1) from Shewanella baltica OS155 was solved at $2.3 \AA$ A resolution (PDB entry $3 \mathrm{dkq}$ ). Buccaneer reported the most complete trace, with $92 \%$ of side chains docked (Fig. 7a). A consensus model was calculated from seven input traces, with the least complete model having $56 \%$ of side chains docked. The consensus model added an 11-residue fragment to the C-terminus and a 13-residue fragment from Met237 to Asn250 and thus partially closed a 20-residue gap (Fig. 7b, cyan). Xpleo was able to fully close the remaining eight-residue gap from Asn249 to Phe257, resulting in a trace with $97 \%$ of residues docked (Fig. $7 c$ ). Fig. $7(d)$ shows the final refined model in green superimposed on the consensus model.

\section{Conclusions}

Xsolve's design to semi-exhaustively and in parallel explore key parameters of the structure-determination process and to utilize multiple software programs increased efficiency and resulted in high-quality traces. For the 36 data sets that we examined in detail, all indexing, phasing and model-building programs resulted in a top trace and furthermore always contributed to a consensus model. This validates Xsolve's approach to run all parameter and software combinations to termination rather than choosing an optimal strategy. A consensus model can provide an optimal starting point for subsequent manual refinement. ConsensusModeler is most effective when input models exhibit variation in completeness and accuracy. Aggressive model-building efforts by some programs, resulting in higher model completeness at the expense of elevated tracing errors, can be offset by a more conservative approach employed by others.

$X$ solve has been instrumental to structure determination at scale, allowing the JCSG to deposit 200 high-quality structures per year in the PDB for the last few years. While parallelism inevitably results in some computational overhead, Xsolve's run-time on each single data set generally does not exceed that of the slowest combination of programs. The model-building stage is the slowest step, ranging from a few hours to several days for large structures. Once completed, the median number of calendar days to refine an initial set of coordinates from Xsolve was only seven. Furthermore, the JCSG's structures

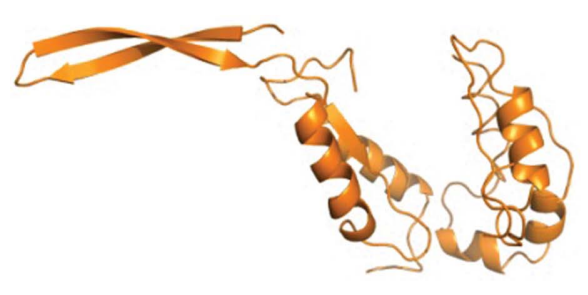

(a)

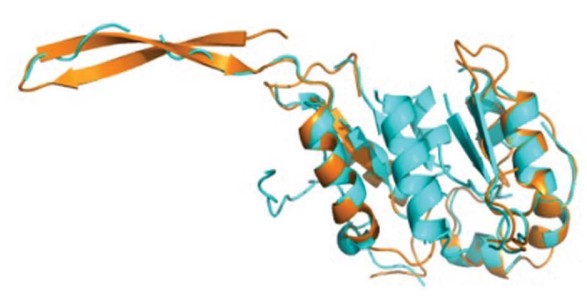

(b)

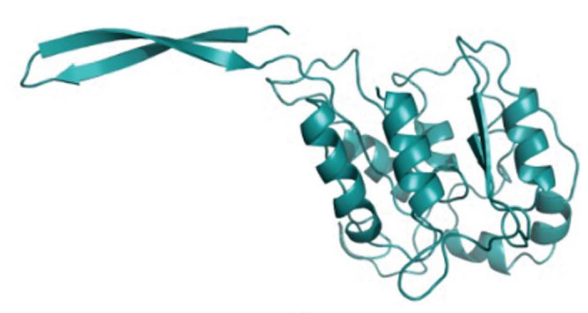

(c)

Figure 6

ConsensusModeler with two input models at $2.8 \AA$ A resolution. (a) The Buccaneer/SHARP/XDS model had $57 \%$ of the sequence docked into the model. (b) The RESOLVE/SHARP/XDS model had $39 \%$ of the sequence docked. (c) The consensus model resulted in $78 \%$ of the sequence docked. 


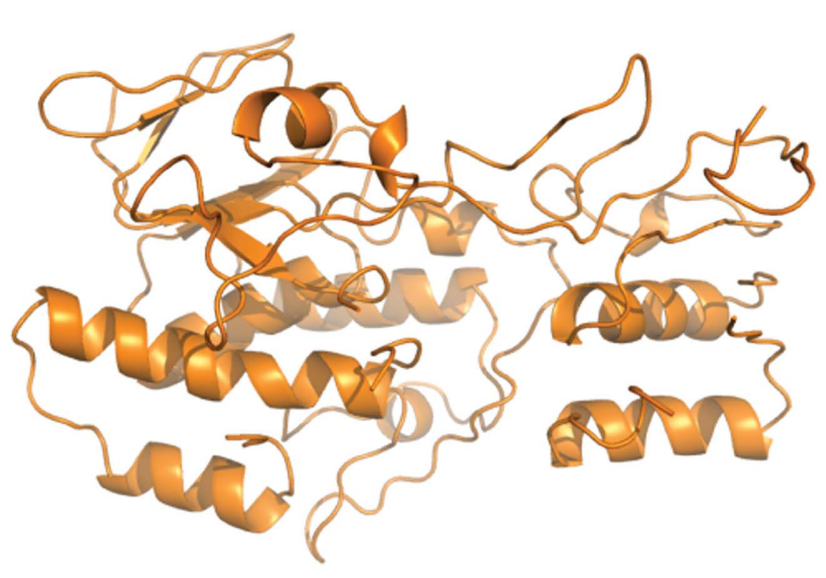

$(a)$

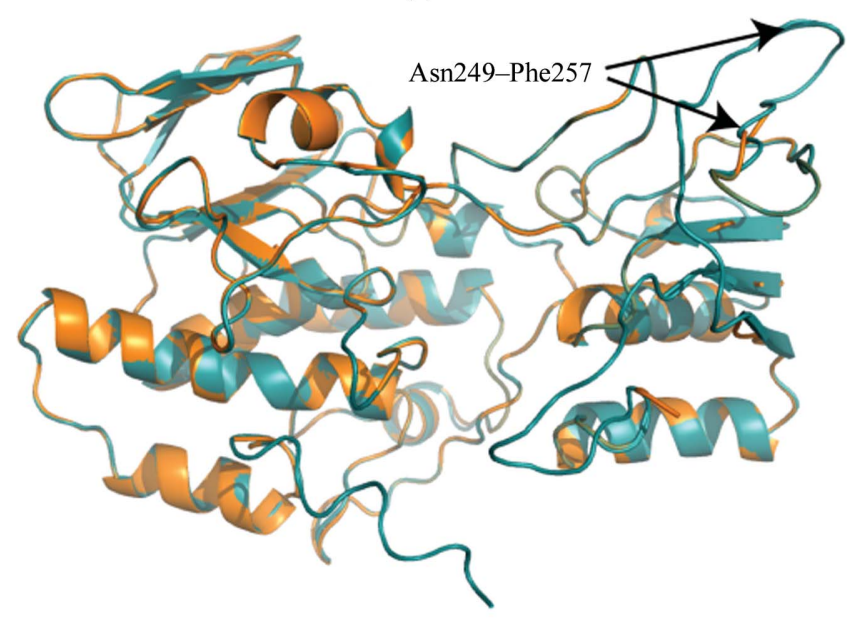

(c)

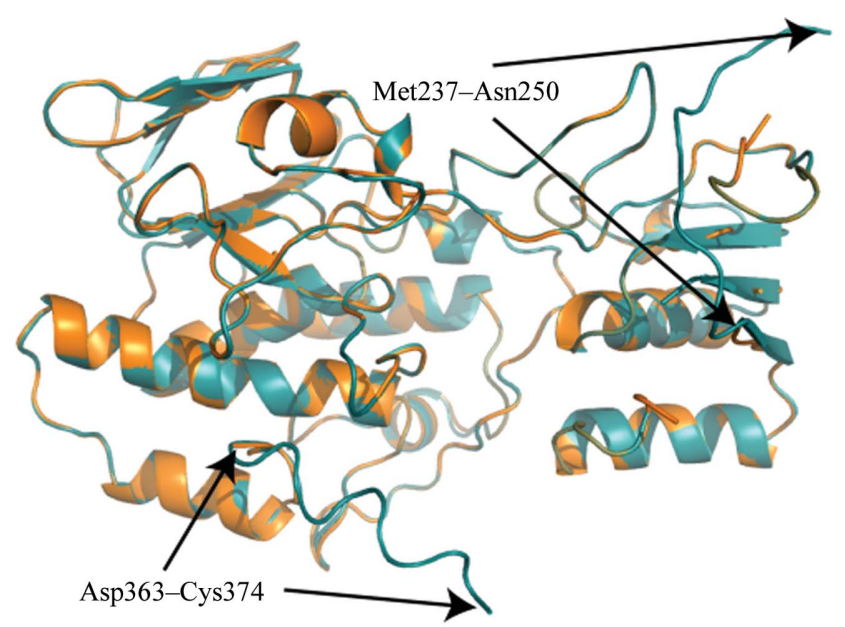

(b)

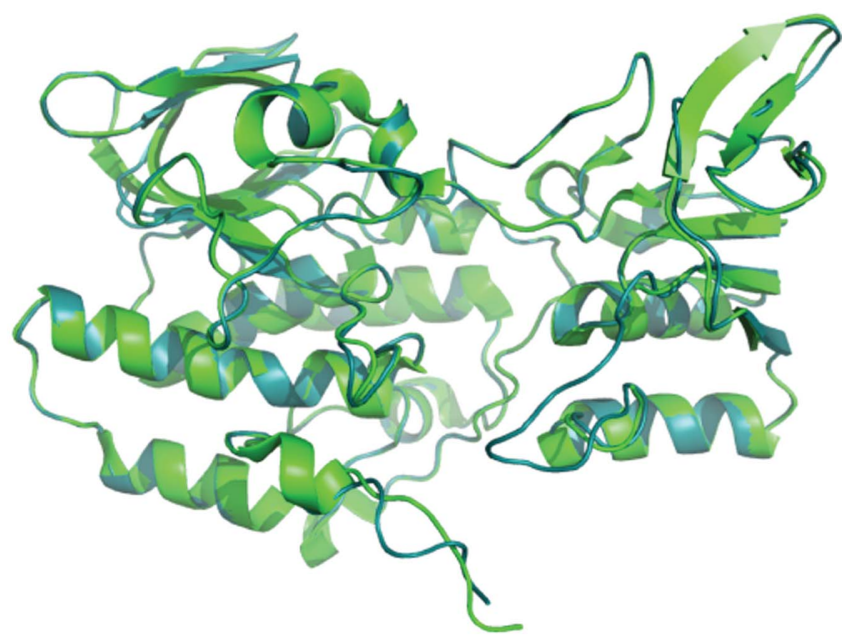

(d)

Figure 7

ConsensusModeler-facilitated Xpleo. (a) The Buccaneer model had $92 \%$ of side chains docked. (b) The consensus model (cyan) calculated from seven input traces, with an 11-residue fragment added to the C-terminus and partially closing a 20-residue fragment from Met237 to Phe257. (c) Xpleo was able to fully close the remaining eight-residue gap from Asn249 to Phe257, resulting in a trace with $97 \%$ of residues docked. $(d)$ The final refined model in green superimposed on the consensus model.

scored highly in an independent broad quality survey (Brown \& Ramaswamy, 2007).

It should be emphasized that all software programs in Xsolve are run with default parameter settings. The results reported in $\$ 3$ for model-building and phasing programs are therefore not representative of those that could be obtained by a skilled crystallographer using the same programs.

A proof-of-concept version of ConsensusModeler was implemented by George Boxer during an internship at SSRL in the summer of 2006. The authors thank all members of the JCSG for their assistance in providing data, suggestions and feedback on structural models. This work was partially supported by NSF grant DMS-0443939. Any opinions, findings and conclusions or recommendations expressed in this paper are those of the authors and do not necessarily reflect the views of the NSF. Test structures used in this work were solved and deposited as part of the JCSG pipeline (http:// www.jcsg.org). The JCSG is funded by NIH Protein Structure Initiative grants P50 GM62411 and U54 GM074898.

\section{References}

Adams, P. D. et al. (2010). Acta Cryst. D66, 213-221.

Bedem, H. van den, Dhanik, A., Latombe, J.-C. \& Deacon, A. M. (2009). Acta Cryst. D65, 1107-1117.

Bedem, H. van den, Lotan, I., Latombe, J.-C. \& Deacon, A. M. (2005). Acta Cryst. D61, 2-13.

Berman, H. M., Henrick, K. \& Nakamura, H. (2003). Nature Struct. Biol. 10, 980.

Brown, E. N. \& Ramaswamy, S. (2007). Acta Cryst. D63, 941-950.

Brünger, A. T. (1992). Nature (London), 355, 472-474.

Cowtan, K. (2000). http://www.ysbl.york.ac.uk/ cowtan/clipper/ clipper.html.

Cowtan, K. (2006). Acta Cryst. D62, 1002-1011.

Fu, Z.-Q., Rose, J. \& Wang, B.-C. (2005). Acta Cryst. D61, 951-959.

Heineman, G. T., Pollice, G. \& Selkow, S. (2008). Algorithms in a Nutshell. Sebastopol, California: O'Reilly Media Inc.

Holton, J. \& Alber, T. (2004). Proc. Natl Acad. Sci. USA, 101, 15371542 . 
Kabsch, W. (2010). Acta Cryst. D66, 125-132.

Kissinger, C. R., Gehlhaar, D. K. \& Fogel, D. B. (1999). Acta Cryst. D55, 484-491.

Krissinel, E. \& Henrick, K. (2004). Acta Cryst. D60, 2256-2268.

Leslie, A. G. W. (1992). Jnt CCP4/ESF-EACBM Newsl. Protein Crystallogr. 26.

Murshudov, G. N., Vagin, A. A. \& Dodson, E. J. (1997). Acta Cryst. D53, 240-255.

Otwinowski, Z. \& Minor, W. (1997). Methods Enzymol. 276, 307-326.

Panjikar, S., Parthasarathy, V., Lamzin, V. S., Weiss, M. S. \& Tucker, P. A. (2005). Acta Cryst. D61, 449-457.

Perrakis, A., Morris, R. M. \& Lamzin, V. S. (1999). Nature Struct. Biol. 6, 458-463.
Schwarzenbacher, R., Godzik, A. \& Jaroszewski, L. (2008). Acta Cryst. D64, 133-140.

Sheldrick, G. M. (2008). Acta Cryst. A64, 112-122.

Smith, T. F. \& Waterman, M. S. (1981). J. Mol. Biol. 147, 195197.

Storoni, L. C., McCoy, A. J. \& Read, R. J. (2004). Acta Cryst. D60, 432-438.

Terwilliger, T. C. (2003). Acta Cryst. D59, 38-44.

Terwilliger, T. C. \& Berendzen, J. (1999). Acta Cryst. D55, 849861.

Vagin, A. \& Teplyakov, A. (2010). Acta Cryst. D66, 22-25.

Vonrhein, C., Blanc, E., Roversi, P. \& Bricogne, G. (2007). Methods Mol. Biol. 364, 215-230. 